
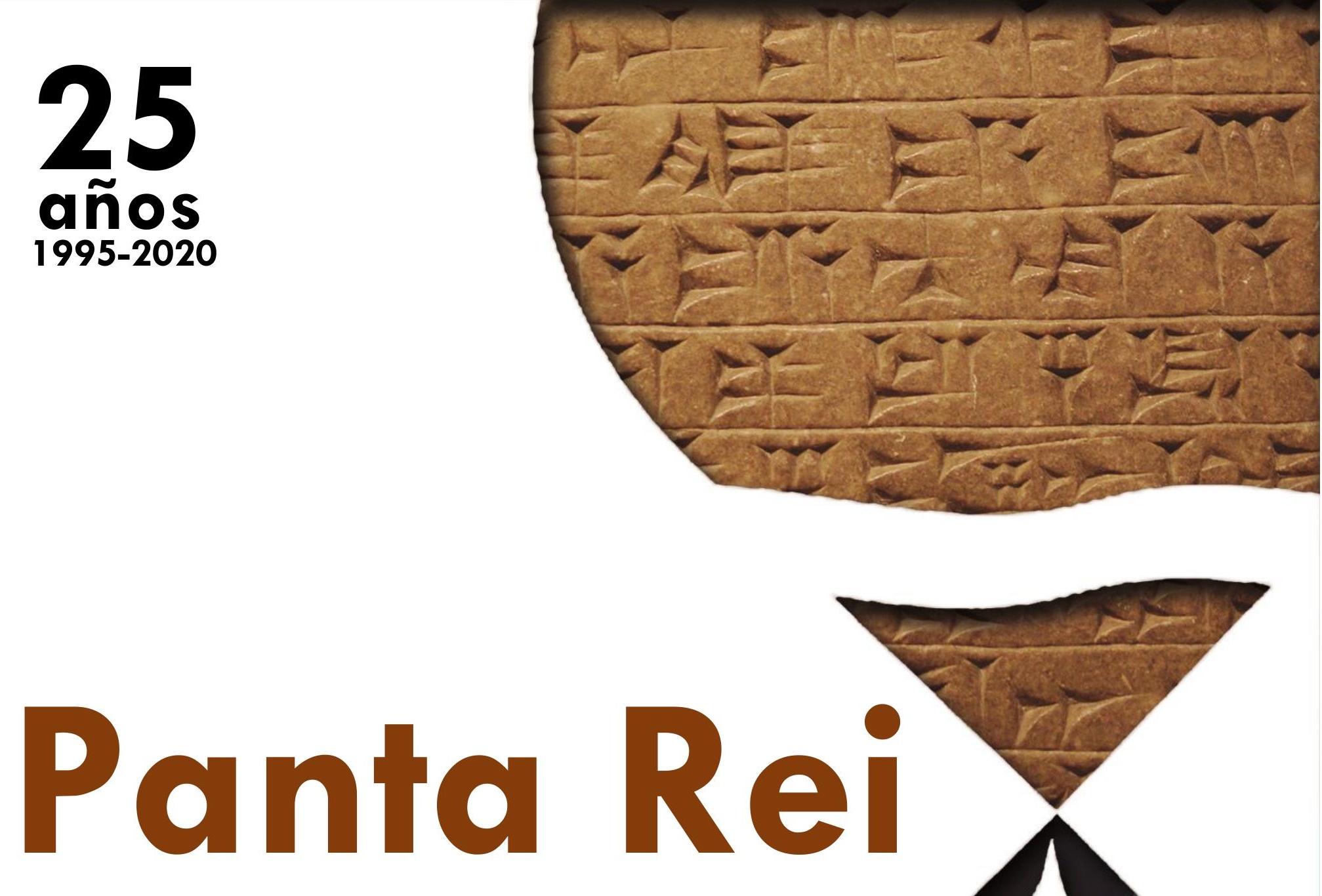

Revista digital de Historia y didáctica de la Historia 


\section{0 (2)}

Revista anual

Fecha de inicio: 1995

Revista Panta Rei. pantarei@um.es

\section{Edita:}

Centro de Estudios del Próximo Oriente y la Antigüedad Tardía - CEPOAT

Edificio Universitario Saavedra Fajardo.

Universidad de Murcia

C/ Actor Isidoro Máiquez, 9

30007 - MURCIA - ESPAÑA

Teléfono: $(+34) 868883890$

cepoat@um.es

Web: www.um.es/cepoat/pantarei

Ediciones de la Universidad de Murcia - EDITUM

Edificio Pleiades. Campus de Espinardo.

Universidad de Murcia

C/ Campus, s/n

30100 - MURCIA - ESPAÑA

Teléfono: $(+34) 868883013$

editum@um.es

Web: https://www.um.es/web/editum/

En portada: Montaje elaborado a partir de fotografía de una inscripción sumeria del British Museum (Wikimedia) (arriba) y fotografía de Miguel Martínez Sánchez (abajo)

Edición 2020 (2)

ISSNe: 2386-8864

ISSN: $1136-2464$
Responsables de los textos: sus autores.

Responsable de la presente edición: Consejo Editorial de Panta Rei.

Depósito legal: MU-966-1995 


\section{CONSEJO DE REDACCIÓN}

Coordinador editorial

Egea Vivancos, Alejandro [Didáctica de las Ciencias Sociales, Universidad de Murcia]

Secretaria

Arias Ferrer, Laura [Didáctica de las Ciencias

Sociales, Universidad de Murcia]

Editores

Jiménez Vialás, Helena [Historia Antigua, Universidad de Murcia]

Martínez Gil, Tània [Didáctica de las Ciencias

Sociales, Universidad de Barcelona]

Meseguer Gil, Antonio José [Historiador, Profesor de

Secundaria]

Ortiz García, Jónatan [Arqueología, Universidad de

Alcalá de Henares]

Romero Molero, Alberto [Arqueología, Universidad

Isabel I]

Sáez Giménez, David Omar [Historiador, Profesor

de Secundaria]

Sáez Rosenkranz, Isidora V. [Didáctica de las

Ciencias Sociales, Universidad de Barcelona]

Sánchez Mondejar, Celso Miguel [Arqueólogo,

Patrimonio Inteligente]

Responsable informático

Martínez García, José Javier [CEPOAT, Universidad de Murcia]

Responsables de traducción y corrección lingüística Martínez Martínez, Cristina [Profesora de Secundaria, Sociedad Española de Lenguas Modernas] Albaladejo Albaladejo, Sara [ISEN-Universidad de Murcia]

\section{CONSEJO ASESOR}

Adroher Auroux, Andrés María [Arqueología, Universidad de Granada]

Albero Muñoz, $M^{a}$ del Mar [H. ${ }^{a}$ del Arte, Universidad de Murcia]

Alia Miranda, Francisco [Historia Contemporánea, UCLM]

Arciniega García, Luis [Historia del Arte, Universidad de Valencia]

Barrio Barrio, Juan Antonio [Historia Medieval,

Universidad de Alicante]

Castellano i Solé, Núria [Egiptología, Schola

Didàctica Activa S.L.]

Chapman, Arthur [History Education, University

College of London, Reino Unido]

Cid López, Rosa María [Historia Antigua, Universidad de Oviedo]

Cobacho López, Ángel [Derecho, Universidad de Murcia]

Cuenca López, José María [Didáctica de las Ciencias Sociales, Universidad de Huelva]
Egea Bruno, Pedro M. ${ }^{a}$ [Historia Contemporánea, Universidad de Murcia]

Feijoo Martínez, Santiago [Arqueología, Consorcio

Ciudad Monumental de Mérida]

García Atienzar, Gabriel [Prehistoria, Universidad de

Alicante]

Ginestí Rosell, Anna [Filología Clásica, Katholische

Universität Eichstätt-Ingolstadt]

González Monfort, Neus [Didáctica de las

Ciencias Sociales, Universidad Autónoma de

Barcelona]

González Soutelo, Silvia [Arqueología, Universidad de Vigo]

Haber Uriarte, María [Prehistoria, Universidad de Murcia]

Hernández de la Fuente, David [Filología Clásica, Universidad Complutense]

Hutson, Scott R. [Anthropology, University of Kentucky, EEUU]

Igual Luis, David [Historia Medieval, UCLM]

Irigoyen López, Antonio [Historia Moderna,

Universidad de Murcia]

Jover Maestre, Francisco Javier [Prehistoria,

Universidad de Alicante]

Mahony, Simon [Digital Humanities, University College of London, Reino Unido]

Marsilla de Pascual, Francisco Reyes [Técnicas

historiográficas, Universidad de Murcia]

Martínez-Burgos García, Palma [H. ${ }^{a}$ del Arte, UCLM]

Mathis, Christian [Didaktik der Geschichte, PH Zürich]

Miralles Maldonado, José Carlos [Filología Clásica,

Universidad de Murcia]

Molina Gómez, José Antonio [Historia Antigua,

Universidad de Murcia]

Mónica Ghirardi [Historia Moderna, Universidad

Nacional de Córdoba, Argentina]

Navarro Espinach, Germán [Historia Medieval,

Universidad de Zaragoza]

Noguera Celdrán, José Miguel [Arqueología,

Universidad de Murcia]

Ortiz Heras, Manuel [Historia Contemporánea, UCLM]

Panzram, Sabine [Historia Antigua, Universität

Hamburg]

Pérez Molina, Miguel Emilio [Filología Clásica,

Universidad de Murcia]

Prados Martínez, Fernando [Arqueología,

Universidad de Alicante]

Sánchez lbáñez, Raquel [Didáctica de las Ciencias

Sociales, Universidad de Murcia]

Sancho Gómez, Miguel Pablo [Educación, UCAM]

Victoria Moreno, Diego [Historia Contemporánea,

UNED]

Vilar García, María José [Historia Contemporánea,

Universidad de Murcia]

Vivas Sainz, Inmaculada [H. ${ }^{a}$ del Arte, UNED]

Zamora López, José Ángel [Próximo Oriente Antiguo,

CCHS-CSIC] 



\section{Índice}

1995-2020. 25 años de Panta Rei

Equipo editorial

La Historia social aplicada a la antigua Mesopotamia: cambios historiográficos y nuevas vías de 9 investigación

Josué Javier Justel Vicente

A Look Back into Ancient Egyptian Linguistic Studies (c. 1995-2019)

Carlos Gracia Zamacona

La infancia en la antigua Mesopotamia: 25 años de investigación

Daniel Justel Vicente

Conflictos armados y su tratamiento en educación. Análisis de la producción científica de los últimos

25 años en la Web of Science

Naiara Vicent, Janire Castrillo, Alex Ibañez-Etxeberria y Leire Albas

La investigación en pensamiento histórico. Un estudio a través de las tesis doctorales de Ciencias

Sociales (1995-2020)

Álvaro Chaparro Sainz, M.a del Mar Felices de la Fuente y Laura Triviño Cabrera

Las narrativas nacionales en la enseñanza y el aprendizaje de la historia: Una revisión sobre los 149 libros de texto y las narrativas de los estudiantes

César López Rodríguez

La historia de las personas: reflexiones desde la historiografía y de la didáctica de las ciencias 167 sociales durante los últimos 25 años

Mariona Massip Sabater, Jordi Castellví Mata y Joan Pagès Blanch †

Panorámica de la trilogía cine, historia y educación en España (1995-2020)

Concha Fuentes-Moreno y Alba Ambrós-Pallarés 



\title{
La infancia en la antigua Mesopotamia: 25 años de investigación
}

\author{
Childhood in Ancient Mesopotamia: 25 Years of Research
}

Recibido: 14/04/2020

Aceptado: 15/07/2020

\section{Resumen}

El artículo plantea un recorrido por las principales investigaciones sobre la infancia en la antigua Mesopotamia. Estos análisis se han desarrollado tradicionalmente de forma paralela a los diferentes estudios sobre la familia en la Antigüedad. Los documentos cuneiformes que nos informan sobre los niños del Próximo Oriente antiguo no son solo numerosos, sino también variados en naturaleza literaria, en procedencia geográfica y cronológica. Las aproximaciones al tema han tenido una marcada evolución historiográfica y se han desarrollado de manera muy especial durante las últimas dos décadas y media. En el artículo se pretende poner en valor la importancia de este campo, centrando especialmente la atención en las investigaciones desarrolladas al respecto durante los últimos 25 años. Finalmente, se señalan brevemente las perspectivas de futuro de una cuestión con un potencial investigador evidente.

\section{Palabras clave}

Historiografía, Historia de Oriente Medio, Familia, Hijos, Comportamiento infantil, Historia antigua.

\begin{abstract}
The article is articulated around the main investigations on childhood in ancient Mesopotamia. These analyses have traditionally been developed in parallel with the different studies on family in Antiquity. Cuneiform documents that inform us about children in the Ancient Near East are not only numerous, but also varied in literary nature, in geographic and chronological provenance. The approaches to the subject have had a marked historiographic evolution and have been specially developed in during the last two and a half decades. The article aims to highlight the importance of this field, focusing in particular on the research carried out in this regard over the last 25 years. Finally, the prospects for the future of an issue with an evident research potential are briefly indicated.
\end{abstract}

\section{Keywords}

Historiography, Middle Eastern History, Family, Children, Infant Behavior, Ancient History.

Para citar este artículo: Justel Vicente, D. (2020). La infancia en la antigua Mesopotamia: 25 años de investigación. Panta Rei: revista digital de Historia y didáctica de la Historia, 43-54. doi: $10.6018 /$ pantarei.445511 


\section{Introducción. Familia e infancia: evolución de la investigación y estado de la cuestión hace 25 años}

Muchos son los estudios y debates que giran en torno al concepto de "familia" en nuestra sociedad moderna occidental. Las aproximaciones a la cuestión son asimismo variadas, y con frecuencia están intrínsecamente relacionadas con posicionamientos concretos y perspectivas (pre-)definidas, sean de carácter político, religioso o de otras índoles. Parece evidente, por tanto, que al abordar el análisis de una realidad tan rica como la familia no debemos contemplar ésta como algo que podamos definir unívocamente. Esta ardua - si no imposible - tarea de adscribir la compleja institución familiar a una categoría única hace que los múltiples acercamientos tengan irremediablemente que partir de una idea: la familia es heterogénea en su esencia. Por otra parte, su innegable valor, crucial en la organización y estructura socioeconómica de nuestra época, está fuera de toda duda. Evidentemente, estas características no son exclusivas de la familia moderna, y los análisis sobre la familia en el pasado se han nutrido también de esta riqueza para afrontar diversas cuestiones, tanto de carácter particular como general.

Los primeros estudios modernos sobre la familia en el pasado se remontan al siglo XIX. Basándose en concienzudas comparaciones relativas al parentesco de sociedades amerindias contemporáneas a su época, y también anteriores, Lewis $\mathrm{H}$. Morgan (1871) propuso una idea de unidad fundamental de la familia humana. Aún así, esta realidad no se propondría como inmutable: como hombre de su tiempo, la fuerte $-y$ ampliamente aceptada en círculos científicos- corriente evolucionista de la época le llevó a contemplar un cierto matiz de progreso en la concepción de la familia. Varias décadas después, Claude Lévi-Strauss (1949), sin incidir en la vertiente evolucionista de Morgan, retomó el concepto de "unidad familiar" a partir de dos conceptos básicos: la filiación y la alianza. Según el antropólogo, esta última facilitó la cohesión social, garantizando el desarrollo de las sociedades antiguas. Este enfoque, netamente estructuralista, se plasma bien en palabras del mismo Lévi-Strauss, cuando afirma que "pour que la societé soit, il ne suffit pas seulement que l'union des sexes et la procréation établissent des liaisons biologiques entre ses membres. II faut aussi qu'en tels ou tels points du tissu social ces liens ne risquent pas de se distendre et de se rompre" (Lévi-Strauss, 1986).

Estos y otros estudios abordaron la realidad de la familia desde diversas perspectivas, si bien las preeminentes fueron la antropología y etnología. Sin embargo, hasta mediados del siglo XX los análisis de la familia en el pasado se limitaron a explicar a un nivel teórico, especialmente desde una óptica estructuralista, las relaciones de parentesco. Así, y debido muchas veces a la falta de fuentes primarias, a su inaccesibilidad, o simplemente por la falta de interés historiográfico, no se atendió con preferencia a otros temas relacionados con el ámbito familiar. Uno de ellos es el que nos ocupa: la naturaleza y función de los niños en las diferentes sociedades del pasado. ¿̇El concepto de "infancia" era natural o socialmente construido? ¿QQué roles específicos tuvieron los menores en sus respectivos ámbitos históricos? ¿Qué percepción social, sentimental o legal tenían los adultos con respecto a ellos? ¿ Hasta qué punto se podía contemplar a un menor como motor económico determinante de un grupo humano? La investigación sobre los niños en el pasado se ha desarrollado especialmente en los últimos 25 años, pero conviene brevemente trazar un esbozo de cómo se ha llegado hasta los estudios actuales.

Solo en 1960 Philippe Ariès abrió esta línea de investigación, con la publicación del pronto célebre L'Enfant et la vie familiale sous l'Ancien Régime. Por vez primera un trabajo histórico 
situaba al niño como objeto preferente de estudio, analizando - algo que tampoco se había hecho hasta la fecha- fuentes históricas realmente variadas: literatura, obras de arte, vestimentas, juegos, pasatiempos, etc. El autor se centró especialmente en la Baja Edad Media, la época pre-industrial y la industrial. Su tesis, incluso debatida hasta nuestra centuria, como en Crawford y Lewis (2008), Brockliss y Montgomery (2010b) o en el artículo periodístico "Farewell to Mini-Me. The flight over when childhood began" (en https://slate.com/culture/2002/03/thefight-over-when-childhood-began.html), apuntaba a que la idea de "infancia" era moderna, y los niños no fueron contemplados en la Edad Media como un ser diferente de los adultos. Eran más bien — siempre según Ariès- proyecciones del adulto, solamente diferenciados de los mayores por capacidad y estatura. El autor denostaba incluso el concepto de "sentimiento" del adulto hacia el menor, por lo que le confería a este último la categoría de una especie de alien, en el sentido latino del término.

Una de las primeras y más duras críticas a Ariès fue la de Lloyd deMause, quien en su edición The History of Childhood (1974a) se aproximó desde la disciplina de la psicología histórica al estudio de la evolución de la infancia (1974b). Ciertamente deMause facilitó por primera vez la publicación de varios análisis referidos a la historia de los niños, contemplándolos como "algo diferente" al adulto. Partió de la premisa de que hasta la fecha los estudios sobre menores eran eminentemente teóricos, y había que tomar con cautela las referencias a niños del pasado. Sirva como ejemplo que los biógrafos oficiales siempre idealizaban la infancia de grandes personalidades, como muestra deMause (1974). Así pues, la idea de "infancia" ya existiría, según deMause, desde la Antigüedad. Su trabajo, aún contando pronto con detractores como Kett (1975), abría nuevas perspectivas en el estudio de la infancia en distintas épocas históricas. DeMause logró en definitiva abrir una vía historiográfica para "hacer hablar" a los niños de distintos períodos, situándolos como sujetos dependientes de los adultos, pero a la vez como objeto de estudio y pertenecientes a una fase de la vida que les era propia. A partir de su trabajo se dieron múltiples aproximaciones en torno a la infancia en el pasado; sobre la evolución historiográfica del concepto de infancia, interesantes son los estudios de Chassaing (2007) y Crawford y Lewis (2008). Los análisis sobre el niño en la Antigüedad no han sido una excepción en este sentido.

\section{El estudio de la infancia en el Próximo Oriente antiguo: estado de la cuestión hace 25 años}

A partir de los postulados y propuestas de deMause (1974a), múltiples y variados han sido los estudios que han tratado la figura del niño en la Antigüedad, como las introducciones a la cuestión de Bouineau (2007b) y Justel (2012b). Las escuelas que primero abordaron la cuestión fueron las británica y francesa. Esta última, a través de la Societé Jean Bodin, organizó, incluso antes de la aparición del libro de deMause, un congreso en Estrasburgo en 1972. El resultado de dicho evento científico fueron cinco volúmenes publicados, todos con título L'Enfant y referidos a períodos distintos o planteados desde perspectivas diversas. Dos de los tomos se dedicaron de manera parcial al niño en distintas civilizaciones antiguas: AA. VV. (1975a) y AA. VV. (1975b). A lo largo de estos estudios se pretendía subrayar los derechos del niño en torno a su vida, educación, integridad física y moral, etc. Los distintos autores abordaron asimismo el ámbito e idiosincrasia de los menores más desprotegidos, como los niños huérfanos o la delincuencia infantil. 
Estos análisis, variados en temática, dieron paso a la paulatina proliferación, aún escasa cuantitativamente, de aproximaciones también diversas. La atención académica al período de infancia en la Antigüedad se desarrolló en los años 80 y 90 del siglo XX especialmente a través de congresos y seminarios cuyas actas eran publicadas o como compendios de análisis que transversalmente trataban varias culturas antiguas. Dos buenos ejemplos se encuentran en Théodoridès, Naster y Ries (1980) y en la primera parte del número 22 de la revista KTEMA (1997).

El estudio de la infancia en la antigua Mesopotamia discurrió historiográficamente por idéntico camino y a similar ritmo que sus homólogos de la Antigüedad. Evidentemente, en ocasiones algunos análisis de los primeros se insertado en obras colectivas de los segundos. En las siguientes líneas nos referiremos diacrónicamente a las aproximaciones que hasta hace 25 años se llevaron a cabo desde la disciplina de la asiriología; esto es, las que se han realizado en torno a la infancia en el Próximo Oriente antiguo a partir de los textos cuneiformes. Tomaremos en consideración, y siempre teniendo como base las fuentes cuneiformes, las referencias a los estudios sobre la infancia en Siria, que arrojan interesantes paralelos con los de Mesopotamia. El presente subapartado no contiene, sin embargo, el análisis historiográfico -asimismo ingente en estudios - de la cuestión para el antiguo Egipto, donde podemos encontrar interesantes trabajos al respecto en Janssen y Janssen (1990), MacDonald (1994) o Feucht (1995).

El primer estudio dedicado a la infancia de la antigua Mesopotamia fue la Tesis doctoral de Michel Gonelle, defendida en París en 1971 y con título La condition juridique de l'enfant en droit suméro-babylonien. En ella el doctorando abordaba cuestiones como la filiación y el estatus del niño, la adopción, las exposiciones infantiles, la educación o los derechos de herencia. Su perspectiva fue eminentemente jurídica, y comprendía documentación datada desde incluso antes de la Tercera Dinastía de Ur (s. XXI a. C.) hasta la época de Hammurabi (s. XVIII a. C.). Para ello escogió documentos paradigmáticos, especialmente procedentes de códigos legislativos (Ešnunna, Lipit-lštar y Hammurabi), aunque también casos de práctica legal, si bien procedentes de un número reducido de archivos (en especial Sippar, Larsa y Nippur). Con todas las limitaciones amén de su condición de inédito-, el estudio de Gonelle abrió nuevas perspectivas en el campo de la infancia en la antigua Mesopotamia, augurando asimismo su potencial debido a la gran cantidad de documentos sin tratar.

El mismo autor participó un año más tarde, en 1972, en el referido congreso L'Enfant, organizado en Estrasburgo por la Societé Jean Bodin. Los resultados referentes a los niños en el mundo cuneiforme fueron aún escasos en número, habida cuenta de que dicho evento planteaba una aproximación a la infancia en diferentes épocas históricas. Sin embargo, quedó patente que la cuestión de la infancia en Mesopotamia se podía analizar tanto tratando documentación de carácter estrictamente legal y procedente de varios corpora, como Gonelle (1975), o profundizando en un solo archivo a partir de otro tipo de textos. Fue este último el caso de Klíma (1975), quien abordó el estatus de los niños basándose en la documentación epistolar de Mari. Aún con todo, las perspectivas planteadas seguían siendo de corte jurídico, y referidas en todo caso al período paleobabilónico.

Un salto cualitativo referente en el campo de estudios sobre infancia en el Próximo Oriente antiguo fue el volumen en francés y holandés L'Enfant dans les civilisations orientales / Het Kind in de Oosterse Beschavingen, editado por Théodorides, Naster y Ries en 1980. Como el propio título 
indica, los quince artículos presentados se centraron geográficamente en Oriente, y solamente dos se referían a un período distinto a la Antigüedad. Así, el mundo cuneiforme estuvo bien representado, con trabajos esta vez sí variados sobre la condición legal de la infancia en época de Ur III y paleobabilónica (Limet), el currículum y formación escolar de los niños a partir de la documentación sumeria (Vanstiphout, Sauren), cuestiones de terminología referida a la infancia en lengua hitita (Lebrun) $\circ$ incluso las primas que las mujeres trabajadoras recibían por el nacimiento de hijos en un contexto aqueménida, a partir de la documentación cuneiforme elamita (Naster).

Las diferentes aproximaciones de la edición de Théodorites et al. (1980) mostraron por primera vez en la historiografía asiriológica el potencial de los estudios sobre infancia en el Próximo Oriente antiguo, y pronto comenzaron a surgir volúmenes de autoría única sobre los niños en la antigua Mesopotamia. Un ejemplo paradigmático fue el estudio de Walter Farber (1989), quien compiló y analizó magistralmente numerosos textos - principalmente sumerios y acadios- de encantamientos en relación con la infancia. Se ponía de relieve de esta manera la importancia de determinados rituales que los mesopotámicos llevaban a cabo para atraer o ahuyentar determinados seres sobrenaturales que podían beneficiar o dañar al bebé recién nacido.

En el mismo año 1989 se abría un interesantísimo tema que ha hecho correr hasta la fecha ríos de tinta: la comparación entre diversos aspectos de los niños en la literatura bíblica y la documentación cuneiforme. La Tesis Doctoral de Joseph Fleishman (1989) daba paso a múltiples estudios y de muy variado carácter sobre el niño en la Biblia y el antiguo Oriente. Evidentemente, la conexión entre ambos mundos había sido planteada hasta entonces en relación a otros muchos temas, pero aspectos como adopciones de niños, ventas infantiles, expósitos, educación, etc., serían abordados con profusión a partir del trabajo de Fleishman, especialmente durante las dos últimas décadas. Es reseñable la labor del mismo Fleishman en este sentido. Siempre desde una perspectiva jurídica comparada entre la Biblia y la documentación cuneiforme, ha publicado numerosos artículos y libros consagrados a la cuestión, entre los que destacan Fleishman (1999, 2000,2001 y 2011 ).

\section{Recientes investigaciones sobre la infancia en la Antigüedad}

Se puede afirmar que el verdadero punto de inflexión en los estudios sobre los niños en la Edad Antigua se dio a finales del siglo XX. Tesis Doctorales, volúmenes en autoría o editados comenzaron a proliferar. Una pequeña prueba de ello son los trabajos de Cohen (1993), Dasen (2003), Ondine Pache (2004), Kunz-Lübcke (2007) ○ Bouineau (2007a), que proponían novedades sobre el tema basándose principalmente - aunque no de forma exclusiva- en fuentes epigráficas. Paralelamente, durante la primera década del siglo XXI la investigación arqueológica complementó dicha visión limitada a las fuentes escritas. Así, en el año 2000 Sofaer Derevenski publicó su afamado Children and Material Culture, que dio lugar a variados trabajos sobre la infancia a partir de registros arqueológicos, como el de Baxter (2004). La mejor prueba de ello en nuestro país es el monográfico de Complutum 21/2 (2010), titulado Infancia y cultura material en arqueología y editado por Margarita Sánchez Romero. En él, varios especialistas nacionales y extranjeros abordaban cómo las fuentes arqueológicas nos podían informar sobre la vida de los niños en el pasado, con cuestiones como la salud y alimentación infantiles, juegos, aprendizajes de técnicas por parte de los menores, etc. 
Fue necesaria la conjunción de varias aproximaciones (sociológicas, etnográficas, textuales, arqueológicas, etc.) para arribar a la "edad madura" de los estudios sobre la infancia en la Antigüedad: en octubre de 2005 tuvo lugar la reunión inaugural de la Society for the Study of Childhood in the Past (SSCIP). Esta asociación nació con la intención de agrupar los altamente diseminados trabajos sobre la infancia en la Antigüedad que se publicaban hasta la fecha (cf. Murphy, 2008, p. 1). Su labor desde entonces ha sido clave en la difusión académica sobre los niños en el pasado: desde 2007 organizan anualmente la Childhood in the Past International Conference, desde 2008 publican la revista Childhood in the Past. An International Journal (hasta el momento doce números) y desde 2010 publican la colección Childhood in the Past Monograph Series (hasta el momento cinco volúmenes). Su labor ha significado un soplo de aire fresco en los estudios sobre la infancia en el pasado, que continúa complementándose con otros análisis más específicos, a partir de fuentes concretas o referidos a períodos más determinados. Un caso paradigmático han sido las aproximaciones realizadas a la infancia en el Próximo Oriente antiguo.

\section{El estudio (moderno) de la infancia en la Mesopotamia antigua: casi 25 años de investigación}

Si bien desde la década de los 70 del siglo XX el tema de la infancia en el Próximo Oriente antiguo había sido tratado (§2), podemos considerar el año 1997 como el inicio de los estudios modernos sobre la cuestión. Fue el momento en el que se generalizaron los análisis sobre diferentes aspectos de los niños en el mundo cuneiforme, principalmente por la edición de nuevos textos y, sobre todo, por la voluntad y actividad de asiriólogos de varios países.

En diciembre de 1997 se organizó en la Maison René Ginouvès de Nanterre la mesa redonda Enfance et éducation dans le Proche-Orient ancien, dirigida por Brigitte Lion, Cécile Michel y Pierre Villard. Publicada en la primera parte de la revista KTÉMA. Civilisations de l'Orient, de la Grèce et de Rome antiques 22 (1997), el repertorio de estudios fue numeroso y diverso en naturaleza. Se analizaron aspectos como las malformaciones infantiles (Cadelli), la educación de reyes durante su infancia (Villard), el papel de las nodrizas (Biga), los niños en un contexto palaciego (Ziegler), la infancia como fase vital (Parayre), la mención a los hijos de comerciantes (Michel), de deportados (Lion) o en particular en textos de períodos concretos (Joannès).

La importancia de la mesa redonda de Nanterre ha sido crucial hasta hoy, puesto que constituyó el "pistoletazo de salida" de los análisis modernos sobre la infancia en el Próximo Oriente antiguo. El estudio sistemático de las fuentes cuneiformes, el trabajo coordinado entre varios asiriólogos y los fondos públicos y privados destinados a sufragar investigaciones y congresos sobre la infancia en la Antigüedad, Mesopotamia incluida (cf. por ejemplo Dasen 2001), han hecho que en el siglo XXI varios sean los investigadores que estudian esta fase de la vida a partir de la documentación cuneiforme.

Una característica fundamental en la investigación de los últimos 25 años sobre la infancia en Mesopotamia ha sido la variedad de archivos, épocas y perspectivas tomados en consideración. Hasta 1997 las aún escasas publicaciones habían abordado el tema basándose fundamentalmente en la documentación legal paleobabilónica. El espectro de corpora, habida cuenta de nuevas publicaciones de textos, se ha ampliado considerablemente, abarcando también la zona asiria, así como varios archivos sirios (especialmente Emar y Ugarit) que nos 
informan en profundidad sobre el tema. La heterogeneidad en los estudios planteados no solo ha sido geográfica, sino también cronológica. Obviamente sin denostar la crucial importancia de la época de Hammurabi, que nos ha provisto con textos únicos relativos a la infancia, la diversidad temporal de los diferentes archivos ha sido asimismo una característica de las últimas dos décadas y media. Así, contamos con estudios que abordan la cuestión a partir de archivos

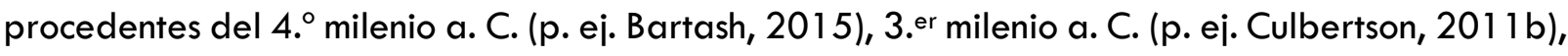
Bronce Reciente (p. ej. Justel, 2018) o 1.er milenio a. C. (p. ej. Wunsch, 2003/2004).

En cuanto a la temática, probablemente la más prolífica óptica durante las últimas dos décadas y media ha sido la jurídica. Contamos con miles de textos antiguos que nos informan sobre distintos aspectos legales de la infancia, como códigos legales, contratos de adopción, ventas de niños, abandonos infantiles, etc. Un primer acercamiento a los niños del Próximo Oriente antiguo, si bien transversal, lo constituyeron los subapartados "Children" (en de la parte dedicada a la familia, §5) dentro de la obra magna editada por Raymond Westbrook, A History of Ancient Near Eastern Law (2003). Además, desde el mencionado trabajo de Fleishman (1989) muchos documentos cuneiformes habían salido a la luz, procedentes de diversos archivos y épocas. Estudios como los de Démare-Lafont (2007), Fleishman (2011), Garroway (2015) o Justel (2018) por fin han situado al niño del mundo cuneiforme como objeto de derecho, pero a la vez como elemento activo en sus respectivas sociedades. Esta visión del menor, incluso como motor económico y protagonista de cambio, se ejemplifica bien en estudios como los de Gehlken (2005) ○ Justel (2015), referidos a las labores desempeñadas por los niños incluso desde un período lactante.

Aún así, durante los últimos 25 años la perspectiva jurídica no ha sido la única contemplada a la hora de aproximarse a la cuestión. De hecho, varios son los trabajos referidos a los niños de la antigua Mesopotamia planteados a partir de textos de carácter religioso o mágico, indisociables ambos conceptos en el Próximo Oriente antiguo. Estudios como el de Stol (2000), Bock (2012) o Flynn (2018) han abordado el tema poniendo justificadamente de relieve la importancia que la descendencia tenía para las sociedades mesopotámicas. Los hijos se contemplaban como un bien para la sociedad, y que un niño llegara a nacer o a curarse de enfermedades durante su infancia era crucial. Contamos para profundizar en estas cuestiones con multitud de textos cuneiformes, como encantamientos, otros sobre el papel de los dioses durante el nacimiento, relatos sobre reyes engendrados por dioses, demonios que actúan contra embarazadas y bebés (sobre Lamaštu, véase Farber, 2014 y Wiggermann, 2000), etc. Varios asiriólogos siguen trabajando en ello, y hay material suficiente para abordar estas $y$ otras cuestiones similares en un futuro próximo.

\section{Conclusiones y perspectivas de futuro}

Las últimas dos décadas y media han constituido cuantitativa y cualitativamente un avance sobremanera considerable en los estudios sobre la infancia en la antigua Mesopotamia. Entes públicos y privados de varios países han sabido valorar la importancia del tema, subvencionando la ejecución de Tesis Doctorales, coloquios, congresos y publicaciones.

Como se ha apuntado, una característica del estudio de la infancia en la antigua Mesopotamia es su potencial. Muchos y variados son los documentos cuneiformes que aún no han sido objeto de estudio conjunto, o al menos han sido tratados solo de manera tangencial. Un análisis sistemático de ellos, tanto aisladamente como comparando varios corpora de diversa naturaleza literaria, 
como pueden ser los de carácter religioso y administrativo, sería bienvenido por la comunidad asiriológica. Asimismo, la comparación de fenómenos relativos a la niñez entre varios archivos, contemporáneos o no, podría ser más tenida en cuenta. Los acercamientos de corte jurídico tampoco han dicho su última palabra. Los nuevos textos que salen a la luz, bien a través de recientes excavaciones arqueológicas, bien por editiones principes de documentos alojados en museos, muestran un entramado legal heterogéneo. Interesante seguirá siendo también, desde perspectivas diversas, la comparación entre estos textos cuneiformes y la documentación de otros ámbitos históricos de la Antigüedad: Egipto, Anatolia, mundos griego y romano, etc.

Sea como fuere, es evidente que los estudios sobre la infancia en el Próximo Oriente antiguo siguen despertando interés, tanto a nivel puramente académico como de difusión general. Uno de los principales atractivos del tema lo constituye el "hacer hablar" a los niños, considerándolos como verdaderos sujetos activos dentro de la sociedad mesopotámica. Es cierto que, tradicionalmente, la historiografía sobre la infancia en el Oriente antiguo contempló al menor como "el otro", alguien sin identidad propia, una proyección del adulto. En los últimos 25 años varios de los estudios referidos han invertido dicha óptica. La aproximación a la infancia se debe hacer situando el sujeto de estudio en el centro del mismo. El material con que contamos para abordar la cuestión es sumamente prolífico y abordable desde varias disciplinas. Estamos seguros de que la producción científica en torno a la infancia en la antigua Mesopotamia, incluso si los textos sobre el tema no crecieran considerablemente en número, seguirá siendo enormemente fructífera al menos durante otros 25 años más.

\section{Bibliografía}

AA. VV. (1975a). L'Enfant. Première partie: Antiquité-Afrique-Asie. Recueils de la Societé Jean Bodin pour l'Histoire comparative des institutions 35. Bruselas: Éditions de la Librairie Encyclopédique.

AA. VV. (1975b). L'Enfant. Cinquième partie: Le droit à l'Éducation. Recueils de la Societé Jean Bodin pour l'Histoire comparative des institutions 39. Bruselas: Éditions de la Librairie Encyclopédique.

Ariès, Ph. (1960). L'Enfant et la vie familiale sous l'Ancien Régime. París: Plon.

Bartash, V. (2015). Children in Institutional Households of Late Uruk Period Mesopotamia. Zeitschrift für Assyriologie und vorderasiatische Archäologie, 105(2), 131-138.

Baxter, J. (2004). Archaeology of Childhood: Children, Gender and Material Culture. Walnut Creek: Alta Mira.

Biga, M.-G. (1997). Enfants et nourrices à Ebla. KTEMA. Civilisations de l'Orient, de la Grèce et de Rome antiques, 22, 35-44.

Bock, U. (2012). "Von seiner Kindheit bis zum Erwachsenenalter". Die Darstellung der Kindheit des Herrschers in mesopotamischen und kleinasiatischen Herrscherinschriften und literarischen Texten. Alter Orient und Altes Testament 383. Múnich: Ugarit-Verlag.

Bouineau, J. (2007a) (ed.). Enfant et Romanité. Analyse comparée de la condition de l'enfant. París: L'Harmattan.

Bouineau, J. (2007b). Éditorial. En Bouineau, J. (ed.), Enfant et Romanité. Analyse comparée de la condition de l'enfant (pp. 13-16). París: L'Harmattan. 
Brockliss, L. y Montgomery, H. (eds.) (2010a). Childhood and Violence in the Western Tradition. Childhood in the Past Monograph Series, volume 1. Oxford: Oxbow Books.

Brockliss, L. y Montgomery, H. (2010b). Introduction. En L. Brockliss y H. Montgomery (eds.), Childhood and Violence in the Western Tradition. Childhood in the Past Monograph Series, volume 1 (pp. 1-18). Oxford: Oxbow Books.

Cadelli, D. (1997). Lorsque l'enfant paraît... malade. KTEMA. Civilisations de l'Orient, de la Grèce et de Rome antiques, 22, 11-33.

Chassaing, J.-F. (2007). L'invention de l'enfance. En J. Bouineau (ed.), Enfant et Romanité. Analyse comparée de la condition de l'enfant (pp. 153-171). París: L'Harmattan.

Cohen, S. J. D. (1993). The Jewish family in Antiquity. Brown Judaic Studies 289. Atlanta: Scholars Press.

Crawford, S. y Lewis, C. (2008). Childhood Studies and the Society for the Study of Childhood in the Past. Childhood in the Past, 1, 5-16.

Culbertson, L. (ed.) (201 1a). Slaves and Households in the Near East, Oriental Institute Seminars 7. Chicago: The Oriental Institute.

Culbertson, L. (2011 b). A Life-Course Approach to Household Slaves in the Late Third Millennium B.C. En L. Culbertson (ed.) Slaves and Households in the Near East, Oriental Institute Seminars 7 (pp. 33-48). Chicago: The Oriental Institute.

Dasen, V. (ed.) (2004). Naissance et petit enfance dans d'Antiquité. Actes du colloque de Fribourg, 28 novembre- 1er décembre 2001. Orbis Biblicus et Orientalis 203. Friburgo: Academic Press.

Démare-Lafont, S. (2007). Réflexions juridiques autour de l'enfance en Mésopotamie. En J. Bouineau (ed.), Enfant et Romanité. Analyse comparée de la condition de l'enfant (pp. 65-85). París: L'Harmattan.

DeMause, Ll. (1974a) (ed.). The History of Childhood. Nueva York: The Psychohistory Press.

DeMause, LI. (1974b). The Evolution of Childhood. En LI. DeMause (ed.), The History of Childhood (pp. 1-73). Nueva York: The Psychohistory Press.

Farber, W. (1989). Schlaf, Kindchen, Schlaf! Mesopotamische Baby-Beschwörungen und -Rituale. Mesopotamian Civilizations 2. Winona Lake: Eisenbrauns.

Farber, W. (2014). Lamaštu. An Edition of the Canonical Series of Lamaštu Incantations and Rituals and Related Texts from the Second and First Millennia B.C. Mesopotamian Civilizations 17. Winona Lake: Eisenbrauns.

Feucht, E. (1995). Das Kind im alten Ägypten. Die Stellung des Kindes in Familie und Gesellschaft nach altägyptischen Texten und Darstellungen. Fráncfort: Campus.

Fleishman, J. (1989). Studies in the Legal Status of Children in the Bible and the Ancient Near East (en hebreo). Tesis Doctoral inedita. Bar Ilan University: Bar Ilan.

Fleishman, J. (1999). Parent and Child in the Ancient Near East and the Bible (en hebreo). Jerusalén: Magnes Press.

Fleishman, J. (2000). On the Legal Relationship between a Father and His Natural Child. Zeitschrift für altorientalische und biblische Rechtsgeschichte, 6, 68-81. 
Fleishman, J. (2001). Who is a Parent? Legal Consequence of Child Maintenance. Zeitschrift für altorientalische und biblische Rechtsgeschichte, 7, 398-402.

Fleishman, J. (201 1). Father-Daughter Relations in Biblical Law. Bethesda: CDL Press.

Flynn, S. W. (2018). Children in Ancient Israel. The Hebrew Bible and Mesopotamia in Comparative Perspective. Oxford: Oxford University Press.

Gehlken, E. (2005). Childhood and youth, work and old age in Babylonia - a statistical analysis. Alter Orient und Altes Testament, 330, 89-120. Münster: Ugarit-Verlag.

Gonelle, M. (1971). La condition juridique de l'enfant en droit suméro-babylonien. Tesis doctoral inédita. Université de Paris, Faculté de Droit et des Sciences Économiques: París.

Gonelle, M. (1975). Le droit à l'éducation, de l'époque de la $3^{e}$ dynastie d'Ur à celle de la dynastie de Hammurabi. En AA. VV. L'Enfant. Cinquième partie: Le droit à l'Éducation. Recueils de la Societé Jean Bodin pour l'Histoire comparative des institutions 39 (pp. 63-77). Bruselas: Éditions de la Librairie Encyclopédique.

Janssen, R. M. y Janssen, J. J. (1990). Growing up in Ancient Egypt. Londres: The Rubicon Press.

Joannès, F. (1997). La mention des enfants dans les textes néo-babyloniens. KTEMA. Civilisations de l'Orient, de la Grèce et de Rome antiques, 22, 119-133.

Justel, D. (2012a) (ed.). Niños en la Antigüedad. Estudios sobre la infancia en el Mediterráneo antiguo. Zaragoza: Prensas de la Universidad de Zaragoza.

Justel, D. (201 2b). El estudio de la infancia en el Mundo Antiguo. En D. Justel (2012a) (ed.). Niños en la Antigüedad. Estudios sobre la infancia en el Mediterráneo antiguo (pp. 15-29). Zaragoza: Prensas de la Universidad de Zaragoza.

Justel, D. (2015). Niños lactantes en las listas de trabajadores forzados de Nippur durante la época mesobabilónica. En A. Bernabé Pajares y J. A. Álvarez Pedrosa (eds.), Orientalística en tiempos de crisis. Actas del VI Congreso Nacional del Centro de Estudios del Próximo Oriente (pp. 233-247). Zaragoza: Libros Pórtico.

Justel, D. (2018). Infancia y legalidad en el Próximo Oriente antiguo durante el Bronce Reciente (ca. 1500-1 100 a. C.). Ancient Near East Monographs 20. Atlanta: SBL Press.

Kett, J. F. (1975). Recensión de DeMause, LI. (1974) (ed.). The History of Childhood. Nueva York: The Psychohistory Press. American Historical Review, 80, 1296.

Klíma, J. (1975). Le status de l'enfant d'après les documents cunéiformes de Mari (première moitié du $2^{\text {ème }}$ millénaire avant J.C.). En AA. VV. L'Enfant. Première partie: Antiquité-AfriqueAsie. Recueils de la Societé Jean Bodin pour l'Histoire comparative des institutions 35 (pp. 119 130). Bruselas: Éditions de la Librairie Encyclopédique.

Kunz-Lübcke, A. (2007). Das Kind in den antiken Kulturen des Mittelmeers. Israel, Ägypten, Griechenland. Neukirchener: Neukirchener Verlag.

Lebrun, R. (1980). Notes sur la terminologie et le status de l'enfant hittite. En A. Théodoridès, P. Naster y J. Ries (dirs.), L'Enfant dans les civilisations orientales / Het Kind in de Oosterse Beschavingen (pp. 43-58). Lovaina: Éditions Peeters.

Lévi-Strauss, C. (1949). Les Structures élémentaires de la parenté. París: Presses Universitaires de France. 
Lévi-Strauss, C. (1986). Préface. En A. Burguière, Ch. Klapisch-Zuber, M. Segalen y F. Zonabend (dirs.). Histoire de la famille. 1. Mondes lointains (pp. 9-16). París: Armand Colin.

Limet, H. (1980). La condition de l'enfant en Mésopotamie autour de l'an 2000 av. J.-C. En A. Théodoridès, P. Naster y J. Ries (dirs.), L'Enfant dans les civilisations orientales / Het Kind in de Oosterse Beschavingen (pp. 5-17). Lovaina: Éditions Peeters.

Lion, B. (1997). Les enfants des familles déportées de Mésopotamie du nord à Mari en ZL 11 '. KTEMA. Civilisations de l'Orient, de la Grèce et de Rome antiques, 22, 109-1 18.

MacDonald, D. N. (1994). Terms for 'Children' in Middle Egyptian: A Sociolinguistic view. Bulletin of the Australian Centre for Egyptology, 5, 53-59.

Morgan, L. H. (1871). Systems of Consanguinity and Affinity of the Human Family. Washington: Smithsonian Institution.

Murphy, E. M. (2008). Editorial. Childhood in the Past. An International Journal, 1, 1-2.

Michel, C. (1997). Les enfants des marchands de Kaniš. KTEMA. Civilisations de l'Orient, de la Grèce et de Rome antiques, 22, 91-108.

Naster, P. (1980). Geboortepremies en Tewerkstelling van Jongeren te Persepolis (van Darius I tot Artaxerxes I). En A. Théodoridès, P. Naster y J. Ries (dirs.), L'Enfant dans les civilisations orientales / Het Kind in de Oosterse Beschavingen (pp. 19-27). Lovaina: Éditions Peeters.

Ondine Pache, C. (2004). Baby and Child Heros in Ancient Greece. Urbana y Chicago: University of Illinois Press.

Parayre, D. (1997). Les âges de la vie dans le répertoire figuratif oriental. KTEMA. Civilisations de l'Orient, de la Grèce et de Rome antiques, 22, 59-89.

Sánchez Romero, M. (2010) (ed.). Infancia y cultura material en arqueología. Complutum $21(2)$. Madrid: Publicaciones Universidad Complutense de Madrid.

Sauren, H. (1980). Het Examenreglement van de Sumerische School. En A. Théodoridès, P. Naster y J. Ries (dirs.), L'Enfant dans les civilisations orientales / Het Kind in de Oosterse Beschavingen (pp. 59-64). Lovaina: Éditions Peeters.

Sofaer Derevenski, J. (2000). Children and material culture. Londres: Routledge.

Stol, M. (2000). Birth in Babylonia and the Bible. Its Mediterranean Setting. Cuneiform Monographs, 14. Groningen: STYX Publications.

Théodoridès, A., Naster, P. y Ries, J. 1980 (dirs.). L'Enfant dans les civilisations orientales / Het Kind in de Oosterse Beschavingen. Lovaina: Éditions Peeters.

Vanstiphout, H. (1980). Over het Vak "Sumerisch” aan de Oudbabylonische Scholen. En A. Théodoridès, P. Naster y J. Ries (dirs.), L'Enfant dans les civilisations orientales / Het Kind in de Oosterse Beschavingen (pp. 29-42). Lovaina: Éditions Peeters.

Villard, P. (1997). L'éducation d'Assurbanipal. KTEMA. Civilisations de l'Orient, de la Grèce et de Rome antiques, 22, 135-149.

Westbrook, R. (ed.) (2003). A History of Ancient Near Eastern Law. Handbook of Oriental Studies, 71-72. Leiden-Boston: Brill. 
Wiggerman, F. A. M. (2000). Lamaštu, Daughter of Anu. A Profile. En Stol, M. Birth in Babylonia and the Bible. Its Mediterranean Setting. Cuneiform Monographs, 14 (pp. 217-252). Groningen: STYX Publications.

Wunsch, C. (2003/2004). Findelkinder und Adoption nach neubabylonischen Quellen, Archiv für Orientforschung, 50, 174-244.

Ziegler, N. (1997). Les enfants du palais. KTEMA. Civilisations de l'Orient, de la Grèce et de Rome antiques, 22, 45-57. 